RACAR : Revue d'art canadienne

Canadian Art Review

\title{
Redonner visage aux gueules cassées. Sculpture et chirurgie plastique pendant et après la Première Guerre mondiale
}

\section{Ada Ackerman}

Volume 41, numéro 1, 2016

URI : https://id.erudit.org/iderudit/1037548ar

DOI : https://doi.org/10.7202/1037548ar

Aller au sommaire du numéro

\section{Éditeur(s)}

UAAC-AAUC (University Art Association of Canada | Association d'art des universités du Canada)

\section{ISSN}

0315-9906 (imprimé)

1918-4778 (numérique)

Découvrir la revue

Citer cet article

Ackerman, A. (2016). Redonner visage aux gueules cassées. Sculpture et chirurgie plastique pendant et après la Première Guerre mondiale. RACAR : Revue d'art canadienne / Canadian Art Review, 41(1), 5-21.

https://doi.org/10.7202/1037548ar
Résumé de l'article

During the First World War, French, British, and US sculptors dedicated their creative practice and knowledge to making masks for soldiers with facial injuries, thus allying art and science in an attempt to restore the most essential aspect of the soldiers' identities. As artistic resources were mobilized to counter the destructive effects of the war, this new kind of sculpture engendered myths and fantasies about the artists' power. This article argues that ultimately, though, the practice of mask-making was used as a strategy that benefitted the preservation of the prevailing economic and social order.
Tous droits réservés (C) UAAC-AAUC (University Art Association of Canada | Association d'art des universités du Canada), 2016
Ce document est protégé par la loi sur le droit d'auteur. L'utilisation des services d'Érudit (y compris la reproduction) est assujettie à sa politique d'utilisation que vous pouvez consulter en ligne.

https://apropos.erudit.org/fr/usagers/politique-dutilisation/ 


\title{
Redonner visage aux gueules cassées. Sculpture et chirurgie plastique pendant et après la Première Guerre mondiale
}

\author{
Ada Ackerman
}

\begin{abstract}
During the First World War, French, British, and US sculptors dedicated their creative practice and knowledge to making masks for soldiers with facial injuries, thus allying art and science in an attempt to restore the most essential aspect of the soldiers' identities. As artistic resources were mobilized to counter the destructive effects of the war, this new kind of sculpture engendered myths and fantasies about the artists' power. This article argues that ultimately, though, the practice of mask-making was used as a strategy that benefitted the preservation of the prevailing economic and social order.
\end{abstract}

Ada Ackerman est chercheuse permanente en histoire de l'art au CNRS (THALIM). Spécialiste du travail de Sergueï Eisenstein (Eisenstein et Daumier, des affinités électives, 2013), elle s'intéresse aux échanges culturels ainsi qu'à l'intermédialité. La présente étude est issue de recherches qu'elle a effectuées pour l'exposition 1917, pour le Centre Pompidou-Metz. -ada.ackerman@cnrs.fr

1. Citons notamment Roy R. Behrens, «The Role of Artists in Ship Camouflage during World War I’, Leonardo, vol. 32, $\mathrm{n}^{\circ} 1,1999$, p. 53-59; Claudia T. Covert, «Art at War: Dazzle Camouflage», Art Documentation: Journal of the Art Libraries Society of North America, vol. 26, $\mathrm{n}^{\circ}$ 2, 2007, p. 50-56; Danielle Delouche, "Cubisme et camouflage», Guerres mondiales et conflits contemporains, $n^{\circ}$ 171, juillet 1993, p. 123-37; André Mare, Cubismeet Camouflage, 19141918, cat. exp., Musée municipal des Beaux-Arts de Bernay, 1998. Cette convergence présumée entre recherches avant-gardistes et tech-
Au sein des études consacrées au rôle des artistes pendant la Première Guerre mondiale, il est souvent question de la rencontre entre les techniques de camouflage modernes et les ressources picturales de l'avant-garde. Ces études explorent, par exemple, les recherches cubistes d'André Mare et d'André Dunoyer de Segonzac, qui participent à la Section de camouflage de l'armée française chargée de masquer les équipements militaires et les points stratégiques, ou encore les expérimentations vorticistes d'Edward Wadsworth mises à profit pour dissimuler les navires de guerre. ${ }^{1}$ Toutefois, la guerre donne aussi naissance à un autre terrain de convergence entre l'art et la science, plus méconnu et plus inattendu.

En effet, la chirurgie plastique du visage visant à réparer les figures ravagées par le conflit connaît alors d'importants développements en France, en Angleterre, en Allemagne ainsi qu'aux États-Unis. ${ }^{2}$ Dans ce cadre, il arrive que des artistes (des peintres mais surtout des sculpteurs) mettent au service des chirurgiens leur science des proportions et des coloris. Toutefois, les blessures sont parfois telles que la chirurgie se retrouve impuissante à les réparer; il faut alors se tourner vers la fabrication de masques destinés à camoufler les visages des mutilés après opération. Interviennent alors des sculpteurs pour concevoir des masques faciaux et parachever ainsi, à l'aide de leur art, le travail du médecin. C'est en France et en Angleterre que cette pratique très spécifique de la sculpture voit le jour.

Ce texte se propose de fournir un aperçu d'ensemble de ces nouvelles relations qui se tissent entre artistes et chirurgiens durant la Première Guerre mondiale, ainsi que dans le contexte de l'immédiat après-guerre. Il s'agira d'observer comment les ressources créatives des artistes sont mises à contribution pour contrer les effets destructeurs de la guerre sur le visage, partie essentielle du corps humain et constitutive de l'identité, et d'examiner les enjeux découlant d'une telle collaboration entre médecins et sculpteurs, parmi lesquels un nombre important de femmes artistes ont œuvré. Tout en soulignant la part de fantasme que cette pratique de fabrication de masques faciaux a pu susciter, ce texte s'attardera sur son rôle dans le maintien d'un ordre économique et social, que le spectacle par trop visible des blessés de la face risquerait de perturber. 
Le conflit de 1914-1918 constitue une rupture sans précédent en termes de violences infligées au corps, initiant le phénomène de «brutalisation» décrit par George Lachmann Mosse. ${ }^{3}$ Parmi les nombreux mutilés qui envahissent alors l'espace et l'imaginaire publics, les blessés de la face ${ }^{4}$ offrent un spectacle pénible inédit, en raison de leur nombre important et de l'ampleur de leurs blessures. En effet, alors que les blessures à la tête n'ont représenté qu'une part marginale des traumatismes subis par les soldats lors des conflits précédents, durant la Première Guerre mondiale, elles deviennent monnaie courante, tant à cause des progrès de l'artillerie moderne-les redoutables éclats d'obus-que de l'enlisement du conflit en une guerre de position, où le stationnement dans les tranchées expose davantage la partie supérieure des corps. On estime à cinq cent mille le nombre de soldats blessés au visage

niques de camouflage est remise en question par Patrick Deccatte dans «Cubisme et camouflage: un mythe de l'histoire de l'art» (2015), article en ligne, http://dejavu.hypotheses.org/2220.

2. Sur l'essor de la chirurgie maxillo-faciale pendant la Première Guerre mondiale, voir Xavier Riaud, Pionniers de la chirurgie maxillo-faciale (1914-1918), Paris, 2010.

3. George Lachmann Mosse, De la Grande Guerre au totalitarisme: la brutalisation des sociétés européennes, Paris, 1999.

4. L'expression «gueules cassées» n'apparaît qu'après le conflit.

5. Sur les blessés de la face, voir Sophie Delaporte, Les Gueules cassées. Les Blessés de la face de la Grande Guerre, Paris, 1996; Karine FerretDussart, «Les blessés de la face pendant la Première Guerre mondiale en France», La Chirurgie maxillo-faciale à travers I'histoire. À propos des collections du Service de santé des armées au Val-de-Grâce, Paris, 2004, p. 167-274; Xavier Tabbagh, "Chirurgie et médecine», in Claire Garnier et Laurent Le Bon (dir.), 1917, cat. exp., Centre Pompidou-Metz, 2012, p. 111-13.

6. David Le Breton, «Défiguration et greffe du visage», in Jacques Mateu, Mathiey Reynier et François Vialla (dir.), Les Assises du corps transformé. Regards croisés sur le visage, Bordeaux, 2009, p. 121-36, ici p. 122.

7. Ibid., p. 125.

8. La Greffe générale, $n^{\circ} 6$, avril 1918 , p. 23.

9. Records of the American Red Cross, 1917-1934, File 942.52, «Studio of Portrait Masks», 1917-1920, National Archives, Washington.

10. Delaporte, Gueules cassées, op. cit., p. 155-56; Suzannah Biernoff, "Shame, Disgust and the Historiography of War», in Claire Pajaczkowska et Ivan Ward (dir.), Shame and Sexuality: Psychoanalysis and Visual Culture, Londres, 2008, p. 217-36. durant le conflit. ${ }^{5}$ Parmi eux, quinze mille survivent à l'issue des hostilités. Pour nombre d'entre eux, le degré de défiguration aurait pu être considérablement réduit si les délais d'acheminement vers les centres de soins avaient été moindres et si les brancardiers n'avaient été trompés par l'aspect impressionnant des lésions, qui les conduisait à en surestimer la gravité et à évacuer en priorité d'autres types de blessés, ayant davantage de chances, selon eux, de survivre.

Au-delà de la souffrance physique qu'elles provoquent, les blessures de la face plongent les soldats dans un véritable désarroi psychologique dans la mesure où elles les privent de leur visage et donc d'un aspect essentiel et constitutif de leur identité. Ainsi que le rappelle David Le Breton, le visage est ce «territoire du corps où s'inscrit la distinction individuelle». ${ }^{6} \mathrm{Par}$ conséquent, note-t-il, la mutilation du visage en tant que totalité, que «gestalt unique», se distingue radicalement de tous les autres types de mutilations étant donné qu'elle a trait à l'être, à l'identité, là où les autres blessures portent atteinte à des fonctions corporelles. ${ }^{7}$

Littéralement dé-figuré, le soldat ne se reconnaît plus; pire, il ne se perçoit même plus comme un être humain. Un blessé anonyme se décrit ainsi en 1918 comme une masse informe et monstrueuse: «on n'est plus qu'une chose sans nom, un amas monstrueux de chairs déchiquetées, de pansements, de pus, de fièvre empaquetés, œuvre d'amour teinte d'horreur par le canon». ${ }^{8}$ Un rapport de la Croix-Rouge américaine daté de 1917 rapporte à ce sujet que «les mutilés au visage sont très sensibles. Ils ont des miroirs de poche qu'ils sortent en toutes occasions, regardant furtivement leur reflet afin de constater à quel point ils sont horribles à voir». ${ }^{9}$

Pour les proches, la mutilation de la face représente une épreuve particulièrement pénible à surmonter: de nombreuses épouses ne peuvent, par exemple, s'empêcher d'éprouver une vive répulsion lorsqu'elles découvrent le nouveau visage de leur mari blessé. ${ }^{10}$ De manière générale, la vision d'un blessé de la face est souvent source de dégoût et d'effroi, comme l'illustre la réaction de ce brancardier, pour lequel un tel visage ne présente plus rien de naturel, ainsi que le suggère son emploi du terme de masque:

Comment? Un éclat d'obus, un seul a pu faire une telle blessure! Oh! Cachez cette face hideuse, cachez-là. Je détourne les yeux, mais j'ai vu et je n'oublierai pas, dussé-je vivre cent ans... J'ai vu un homme qui, à la place du visage, avait un trou sanglant. Plus de nez, 
À cet égard, le personnel soignant des hôpitaux se confronte à un problème inédit et éprouvant bien décrit par le caporal Ward Muir, en service au Troisième Hôpital Général de Londres: comment un soignant peut-il prétendre soutenir son patient et lui transmettre sa sympathie alors qu'il a peur de poser les yeux sur lui et qu'il redoute encore davantage de croiser son regard? En effet, le soignant sait que son expression horrifiée, impossible à réprimer, révèlerait au patient de manière indubitable à quel point l'apparence de "gargouille brisée» de ce dernier est insoutenable. ${ }^{12}$ On imagine le drame psychologique que représente, pour les blessés de la face, une telle impossibilité de circulation des regards, qui les prive ainsi d'un échange humain primordial.

\section{La réparation d'un visage: une tâche artistique}

Face à ces blessés d'un type particulier, la question de leur prise en charge se pose très rapidement, dès les premières années du conflit. Comment leur rendre une apparence acceptable? Certains chirurgiens se sentent dépassés par l'ampleur de la tâche. Par exemple, dans le rapport mensuel qu'il produit en octobre 1917, Dr Lemaître explique que:

Lorsqu'on pénètre dans un centre de chirurgie maxillo-faciale, ce qui fixe l'attention, ce sont les effroyables délabrements de la face; aussi peut-on être saisi d'épouvante au regard de ces blessures et quand la sensibilité a cédé à la réflexion, quand l'homme fait place au chirurgien, celui-ci se demande comment il va pouvoir réparer de tels délabrements. ${ }^{13}$

De nombreuses techniques de chirurgie maxillo-faciale reconstructrice et notamment de greffes sont alors pratiquées, à l'instigation de chirurgiens pionniers comme les Français Léon Dufourmentel, Hippolyte Morestin, Émile-Jules Moure et Pierre Sébileau, les Britanniques Harold Gillies et William Kelsey Fry, les États-Uniens Varaztad Hovhannes Kazanjian, Robert Ivy et Vilray Blair, ainsi que les Allemands Georg Axhausen, Johannes Esser et Jacques Joseph, ${ }^{14}$ qui militent pour que soit reconnue l'importance de ces opérations, tant d'un point de vue physiologique que psychologique et social. Leurs méthodes, à un stade encore souvent expérimental, s'effectuent à l'aide de multiples prothèses, d'appareils de maintien et de moules. ${ }^{15}$

11. Bernard Lafont, Au Ciel de Verdun, Paris, 1918, p. 10-11.

12. Ward Muir, «The Men with the New Faces», The Nineteenth Century, octobre 1917, p. 746-53, ici p. 746 .

13. Dr Lemaître, «Rapport mensuel d'octobre 1917", Archives du service de santé des armées, Valde-Grâce, carton 124, cité par Delaporte, Gueules cassées, op. cit., p. 85.

14. Leurs innovations et trajectoires sont détaillées dans Riaud, Pionniers, op. cit.

15. Sur ces différentes méthodes, voir Delaporte, Gueules cassées, op. cit., p. 88-120. Dans la plupart des cas, le processus s'accompagne de souffrances intenses, s'apparentant à une véritable torture. Par exemple, pour éviter la constriction maxillaire, la méthode dite «du sac» prévoit que le patient tienne entre ses dents un crochet lesté d'un poids pouvant aller jusqu'à trois kilogrammes. Mais les blessés de la face semblent généralement prêts à tout endurer pour pouvoir recouvrer une apparence humaine et mettent tous leurs espoirs entre les mains des chirurgiens. De nombreuses brochures encouragent d'ailleurs ces espérances, en vantant les prouesses des chirurgiens à l'aide d'une rhétorique visuelle opposant de manière dramatique et spectaculaire photographies du mutilé avant et après opération.

Dans ce cadre, il n'est pas rare que le travail du chirurgien soit perçu comme celui d'un artiste. Au sujet des réalisations de Léon Dufourmentel, 


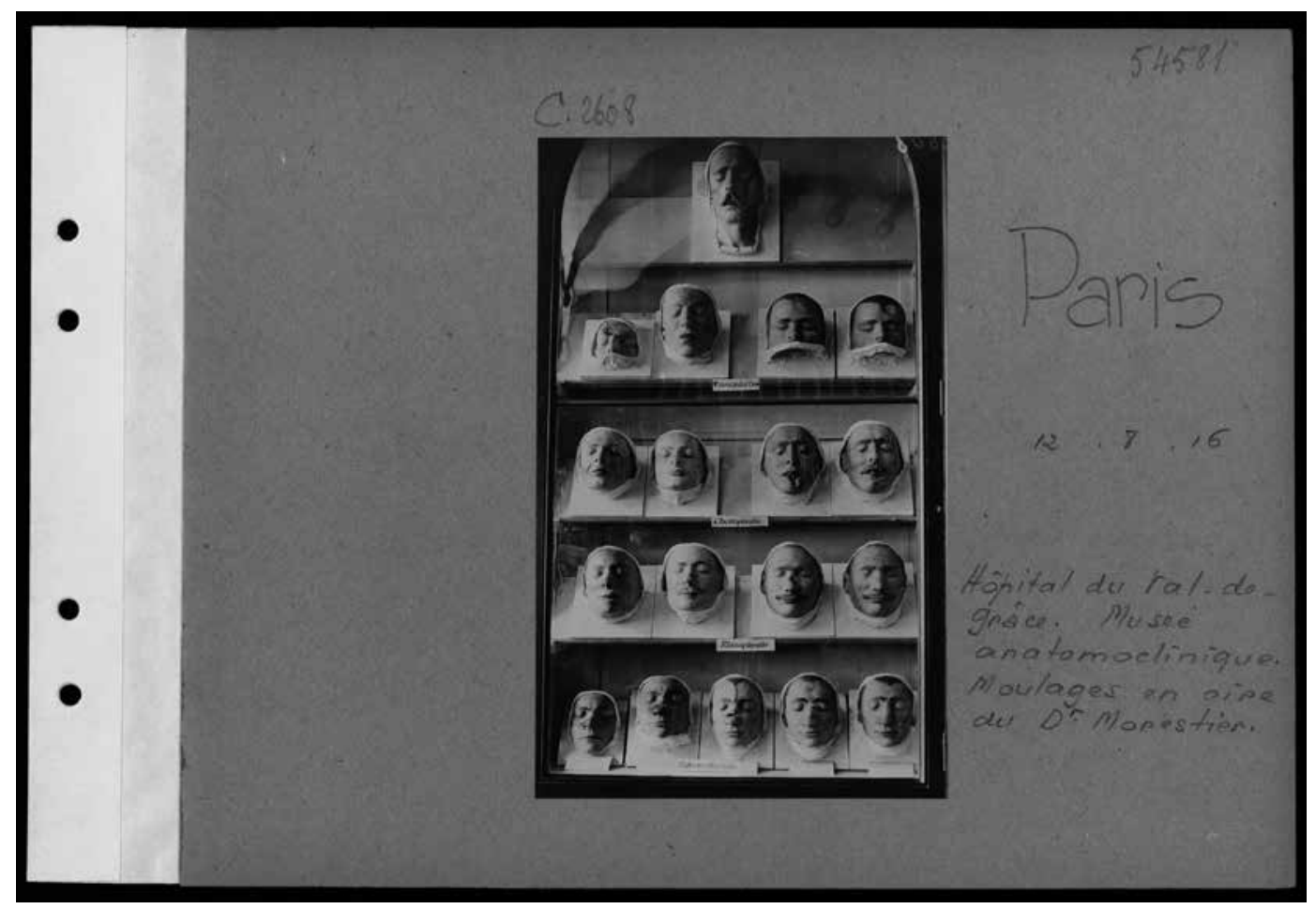

16. Henriette Rémi, Hommes sans visage, Lausanne, 1942, p. 70

17. Léon Dufourmentel, Introduction à l'art de la chirurgie constructive. Essai sur l'art de la chirurgie, Paris, 1946, p. 140.

18. John-Paul Bogart et Martin du Louvre, The Plaster Mask as Remedy. Jane Poupelet and Robert Wlérick's Work for Léon Dufourmentel, non publié. Je remercie Jonathan Zimmerman de m'avoir communiqué ce texte.

19. Ancienne abbaye bénédictine transformée en hôpital militaire en 1793, le Val-de-Grâce est dédié à la médecine militaire. Devenant le Service de santé des armées en 1850 , le complexe héberge en plus de l'hôpital, une bibliothèque spécialisée ainsi qu'un centre d'enseignement. Il comprend également un cabinet d'anatomie qui s'enrichit au fil de différents legs et qui représente l'ancêtre du Musée du Service de Santé des Armées, créé en 1850 et communément appelé Musée du Val-de-Grâce. Le musée ouvre officiellement pendant la Première Guerre mondiale, en 1916, avec pour mission de présenter au public documents et archives relatifs à la guerre. L'hôpital militaire du Val-de-Grâce est l'un des premiers établissements à prendre l'infirmière Henriette Rémi recourt à une métaphore sculpturale, qui lui permet d'insister sur son haut degré d'exigence: «c'est un tailleur de pierre, il ne veut pas montrer la corniche inachevée». ${ }^{16}$ Dufourmentel se réclamera d'ailleurs lui-même de ce paradigme artistique dans L'Essai surl'art de la chirurgie (1946), lorsqu'il déclare, dans une formule aussi lapidaire qu'oxymoristique, que "conserver, c'est créer». ${ }^{17}$

La sensibilité artistique mobilisée par Dufourmentel dans son travail se traduit par sa décision, pionnière, de faire appel à des artistes professionnels pour l'aider dans sa tâche. Les sculpteurs Jane Poupelet et Robert Wlérick réalisent ainsi pour lui différents moulages en plâtre et en cire de ses patients afin d'enregistrer les progrès des opérations et de constituer ainsi un matériau documentaire, dans la lignée des cires anatomiques (céroplastique) popularisées par l'abbé Gaetano Giulio Zummo, dit Zumbo, à partir du XvII ${ }^{\mathrm{e}}$ siècle. ${ }^{18}$ Ces moulages requièrent de véritables compétences artistiques, puisqu'il est nécessaire de maîtriser l'art du portrait et la science des proportions, sans oublier celle des coloris. Les contemporains s'y montrent sensibles, comme le révèle cette critique de Léo Claretie publiée dans le quotidien L'Événement et rédigée à l'occasion de l'ouverture en 1916 du Musée du Val-de-Grâce à Paris, destiné à présenter au public les efforts déployés pendant le conflit par le Service de santé aux armées. ${ }^{19}$ | fig. 1 | L'auteury insiste autant sur l'efficacité de la chirurgie faciale réparatrice que sur la force plastique des moulages exposés:

Le Musée du Val-de-Grâce racontera l'histoire de la guerre à travers les souffrances et leurs remèdes. Les objets de la section de chirurgie anatomo-pathologique moulés par de 
Figure 1. (Ci-contre) Hôpital du Val-de-Grâce. Musée anatomoclinique. Moulages en cire. Collection Morestin, 1916. Collection Bibliothèque de documentation internationale contemporaine.

Figure 2. Henry Tonks, Portrait of Walter Ashworth, 1916-1918, pastel sur papier, $27.3 \times 20.9 \mathrm{~cm}$.

Royal College of Surgeons of England (RCSSC/P 569.50). Photo: (C) The Hunterian Museum at the Royal College of Surgeons. en charge les blessés de la face. Stéphane Chaumont, Contribution à I'histoire del'hôpital du Val-de-Grâceet de ses pharmaciens chefs. Du champ de bataille au champ des grandes découvertes, thèse soutenue en 2013 pour obtenir le titre de docteur en pharmacie, Université Paris-Descartes; Maurice Bazot (dir.), Le Val-de-Grâce, enseignementet culture, Paris, 2004.

20. Léo Claretie, «Le Génie de la Pitié», L'Événement, 12 mai 1916 [nous soulignons]. La collection actuelle du musée du Val-de-Grâce comprend toujours ces moulages, à raison d'environ 1200 pièces. Ce fonds est composé de moulages provenant notamment des services des chirurgiens faciaux Léon Dufourmentel, Hippolyte Morestin, Émile Moure et Pierre Sébileau. Sur les collections du Val-de-Grâce, voir Ferret-Dussart, Chirurgie maxillofaciale, op. cit.

21. Surl'art de Tonks, voir Suzannah Biernoff, «Flesh Poems: Henry Tonks and the Art of Surgery", Visual Culture in Britain, vol. $11, \mathrm{n}^{\circ} 1$, 2010, p. 25-47; Samuel J.J. Alberti, Suzannah Biernoff et coll., War, Art and Surgery, Londres, 2014.

22. Sur ce paradoxe, voir Emma Chambers, «Fragmented Identities: Reading Subjectivity in Henry Tonks's Surgical Portraits», Art History, vol. $32, n^{\circ} 3,2009$, p. 578-607, ici p. 587.

23. Suzannah Biernoff, "The Face of War», in Andrei Pop et Mechtild Widrich (dir.), Ugliness: The Non-Beautiful in Art and Theory, Londres, 2013, p. 38.

24. Lettre à D.S. McColl, 1916, citée par Joseph Hone, The Life of Henry Tonks, Londres, 1939, p. 127.

25. Lady Kennet [Kathleen Scott], Self-Portrait as an Artist, Londres, 1951, p. 168.

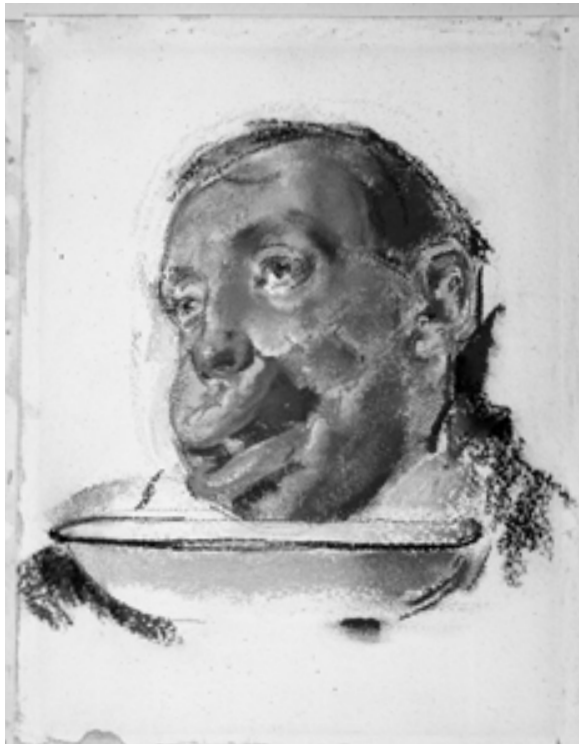

grands artistes offrent le spectacle impressionnant d'un visage broyé confronté au visage réparé. ${ }^{20}$

On observe un phénomène similaire en Grande-Bretagne, où, à l'instar de Dufourmentel, le chirurgien facial Harold Gillies décide de s'assurer le concours d'un artiste professionnel, le peintre Henry Tonks, afin de fixer l'ensemble des processus de son travail avec les mutilés. Tonks, dont le style néo-impressionniste a déjà alors été bien remarqué, a reçu une formation médicale, ce qui en fait un artiste de choix pour la mission que lui assigne Gillies. Désireux de contribuer à l'effort de guerre britannique, Tonks réalise ainsi soixante-douze portraits de mutilés de la face suivis par Gillies, que l'on peut aujourd'hui contempler au Royal Surgeon College of England. ${ }^{21}$ Il y mobilise sa science du pastel et du dégradé pour enregistrer l'évolution des blessures et l'efficacité des traitements, instaurant une équivalence troublante entre la délicatesse inhérente au pastel et la violence faite à la chair. ${ }^{22} \mid$ fig. 2 | Comme le note Suzannah Biernoff, il s'agit d'œuvres au statut insolite et hybride, se trouvant à la croisée entre réalisations documentaires et portraits artistiques. ${ }^{23}$

En effet, bien qu'il assigne à ses croquis une visée avant tout clinique, Tonks insiste sur leur dimension artistique, dans la mesure où, selon lui, ses modèles blessés présentent une beauté insolite, qu'il rattache à l'esthétique classique du fragment: «j'ai fait un dessin l'autre jour d'un jeune homme au visage plutôt classique qui s'apparentait totalement à une tête grecque vivante abîmée, dont le nez aurait volé en éclats». ${ }^{24}$ L'une des émules de Tonks, la sculptrice Kathleen Scott, embauchée par Gillies sur la recommandation de Tonks, recourt elle aussi au paradigme de l'antique pour décrire son travail avec les mutilés. Son propos s'inscrit totalement à contre-courant des discours de l'époque sur la difformité et l'horreur des blessés de la face: «j'ai travaillé sur un homme au visage magnifique et sans nez. Ces hommes sans nez sont très beaux, comme des marbres antiques». ${ }^{25}$ 
Nul doute, comme le suggère Sarah Crellin, que cette conversion de l'horreur en beauté révèle un mécanisme psychologique de la part de l'artiste pour atténuer la pénibilité de sa tâche, consistant à observer et à représenter ces hommes au visage perturbant ${ }^{26}$-le terme de beau étant probablement à entendre ici au sens de matériau intéressant et original, du point de vue de l'artiste. De même, en comparant le cas des mutilés à des marbres grecs antiques, à des ruines dont la spécificité et l'attrait résident précisément dans leur aspect partiel et lacunaire, il s'agit de ramener aux catégories connues du classique des personnes dont l'aspect défie alors l'entendement ainsi que les normes; l'artiste cherche bel et bien à neutraliser et à mettre à distance l'effroi que provoque en lui la vision de ces visages mutilés.

\section{Le camouflage facial: I'artiste à la relève du chirurgien. Enjeux et fantasmes}

Lesculpteur démiurge. Si les artistes sont bel et bien mobilisés aux côtés des chirurgiens dans le cadre d'opérations faciales réparatrices, qu'il s'agisse d'en illustrer le déroulement ou pour témoigner de leur efficacité, il arrive bien souvent que ces opérations ne suffisent pas à rendre à un patient son visage. C'est alors qu'intervient de nouveau l'artiste, qui prend désormais la relève du chirurgien. Comme l'exprime bien le sculpteur britannique Francis Derwent Wood:

Mon travail commence là où le travail du chirurgien s'achève. Lorsque le chirurgien a fait tout ce qui était en son pouvoir [...], je mets à contribution tout le savoir que j'ai la chance de posséder en tant que sculpteur pour tenter de redonner autant que possible à un visage humain l'aspect antérieur à sa blessure. ${ }^{27}$

En effet, dès la fin 1915, Wood, un sculpteur de la Royal Academy d'Angleterre spécialisé jusque-là dans la réalisation de bustes et de sculptures décoratives inspirés par la statuaire antique, obtient de l'armée britannique l'autorisation d'ouvrir au sein du Troisième Hôpital Général de Londres un «Département des masques pour les défigurations faciales», rapidement surnommé le Tin Noses Shop. ${ }^{28}$ Celui-ci se donne pour objectif de fournir des prothèses et des masques faciaux aux mutilés dont les visages ne peuvent être reconstruits de façon satisfaisante par la chirurgie et dont les blessures doivent être par conséquent camouflées. Si leur fonction consiste essentiellement à procurer aux blessés une enveloppe protectrice vis-à-vis des regards, il s'agit aussi concrètement, dans les cas les plus graves, d'éviter d'exposer les lésions et organes au froid, à la chaleur ainsi qu'au bruit. ${ }^{29}$

Faisant preuve de perfectionnisme acharné et travaillant avec une équipe réduite de sculpteurs-Frederick Wilcoxson, William Bateman Fagan et Thomas Humphrey Paget-ainsi qu'avec un mouleur et un fondeur, Wood

26. Sarah Crellin, «Hollow Men: Francis Derwent Wood's Masks and Memorials, 1915-1925", The Sculpture Journal, t. 6, 2001, p. 75-88.

27. Francis Derwent Wood, "Masks for Facial Wounds», The Lancet, 23 juin 1917, p. 949-51.

28. Sur l'atelier de masques de Wood, voir Crellin, «Hollow Men», op. cit.

29. Lettie Gavin, American Women in World Warl. They Also Served, Niwot, CO, 1997, p. 196. réalise pour chacun de ses clients un premier moulage à partir du visage défiguré. Puis il en comble les parties manquantes avec de la pâte à modeler, de manière à restituer l'apparence du visage avant sa mutilation. Pour cela, il s'aide de photographies ou, lorsqu'elles font défaut, de son imagination. Il exécute alors un moulage en cire des parties manquantes, qu'il plonge ensuite dans un bain de cuivre, avant de les peindre de couleur chair, de façon à faire illusion autant que possible. Il en dote certains de poils, de cheveux ou de cils avant de leur ajouter des fils de fer pour qu'ils puissent être attachés. C'est ainsi qu'on trouve dans l'atelier de Wood toutes sortes de substituts aux différentes parties du visage: oreilles, yeux, nez, fronts, etc. | fig. 3 | 
Figure 3. Horace Nicholls, Repairing War's Ravages: Renovating Facial Injuries. Various plates and attachments in different stages of completion. Londres, Imperial Wa Museum, (C) IWM (Q30460).

Figure 4. Horace Nicholls, The Development of Plastic Surgery during the First World War: The Work of Francis Dervent Wood at 3rd General Hospital, Wandsworth, London. Londres, Imperial War Museum, (C IWM (Q 30457).
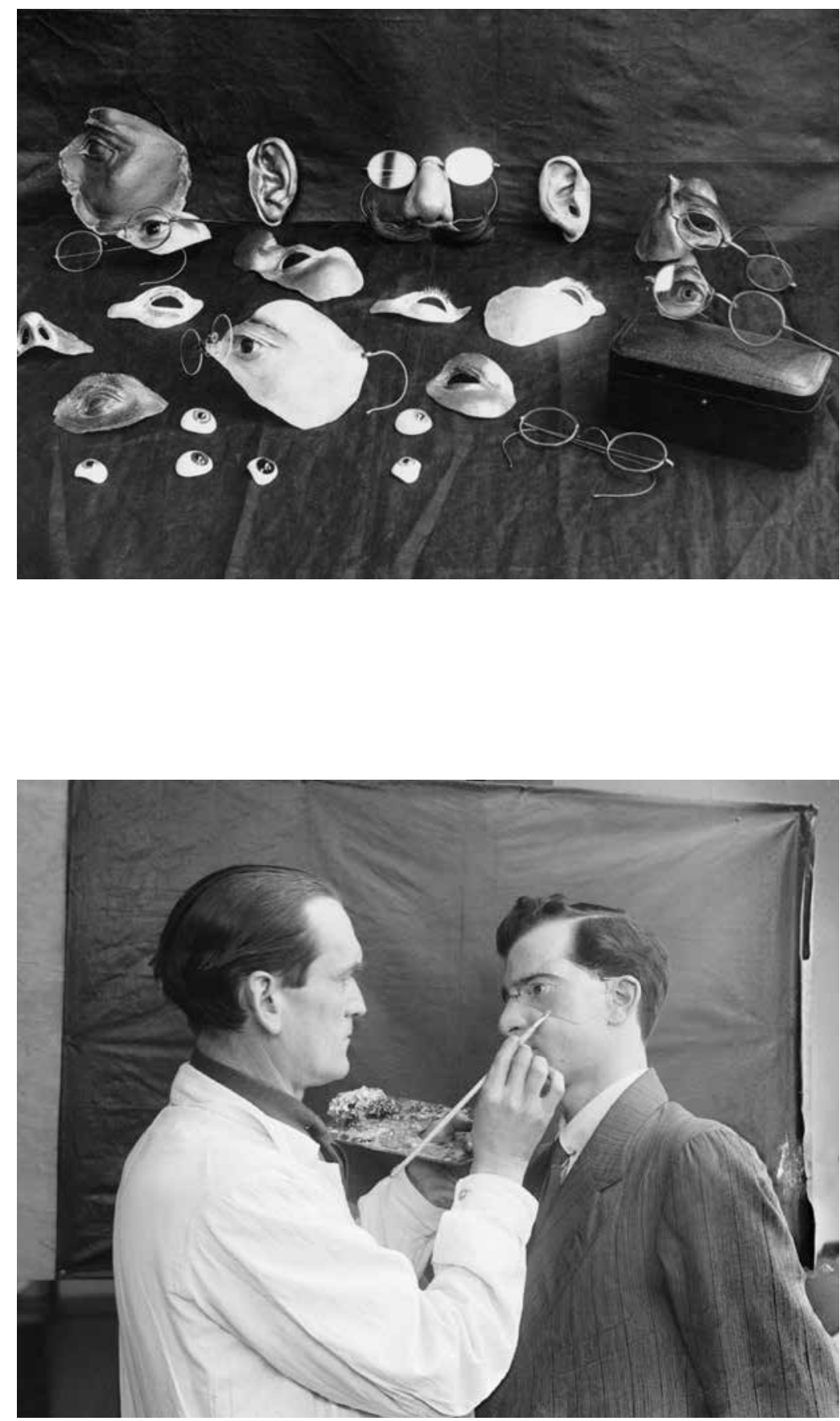
Comme le note Ward Muir, le succès d'une telle entreprise repose entièrement sur les compétences proprement artistiques du personnel du Tin Noses Shop, soulignant à cet égard le caractère inédit de l'entreprise dans l'histoire de la médecine:

Le Département des masques pour les défigurations faciales, aux caractéristiques si intensément pratiques et matérielles, n'aurait pu évoluer, ni même être dirigé par quelqu'un d'autre qu'un sculpteur. Ces plaques de métal peint à la forme curieuse ne se présentent peut-être pas de manière ostensible comme de la pure et simple sculpture. Mais elles s'appuient sur une formule et sur une pratique de la sculpture et sont issues de l'agilité de la main du sculpteur [...]. On ne peut établir aucun parallèle entre le Département des masques pour les défigurations faciales et les autres secteurs de I'hôpital militaire. ${ }^{30}$

La virtuosité et le soin dont Francis Derwent Wood et ses émules font preuve dans cet usage très spécifique de leur art réactive et remet en circulation l'un des mythes récurrents de l'histoire de l'art, à savoir le mythe de Pygmalion. La nouveauté en la matière réside précisément dans la proximité du sculpteur avec le chirurgien, auquel il se substitue et dont il récupère l'aura, en tant qu'être potentiellement capable, par ses connaissances rationnelles, de créer la vie ex nihilo-le mythe de Frankenstein se télescopant ici avec celui de Pygmalion. Ce point est bien illustré par le témoignage de Nellie $\mathrm{N}$. Barnes, une médecin américaine volontaire dans l'armée française. Après avoir visité à Paris l'atelier de fabrication de masques de sa compatriote Anna Coleman Ladd, qui s'inspire de celui de Wood, elle souligne que sa force réside dans cette alliance entre l'art et la science: «Nous nous émerveillons, avec une grande gratitude, de cet art scientifique qui peut redonner la vie à ces victimes innocentes. ${ }^{31}$ Il n'est pas anodin à cet égard que Barnes emploie ici l'expression «redonner la vie» : il s'agit, bien entendu, de la possibilité, pour les patients, de revenir à leur vie antérieure au conflit et à leur blessure. Il faut aussi entendre cette expression de manière littérale, le sculpteur se retrouvant investi d'un pouvoir d'animation.

On observe dès lors, dans la célébration du travail des sculpteurs à l'égard des soldats mutilés, une tendance à les présenter comme véritablement capables de transmuer des masques de cuivre en chair vivante. Celle-ci s'incarne de manière exemplaire dans une photographie d'Horace Nicholls mettant en scène Francis Derwent Wood en train de fabriquer un masque pour un patient. | fig. 4 | Cette photographie fait partie d'une série intitulée Repairing War's Ravages: Renovating Facial Injuries, que la Wellington House, le bureau de propagande britannique, avait commandée à Nicholls, un photographe britannique officiellement employé par l'armée. ${ }^{32}$ S'appuyant sur une mise en

30. Muir, «The Men with the New Faces», op. cit., p. 753.

31. Autobiographie de Nellie N. Barnes, Pope County Historical Society, Glenwood, cité par Gavin, American Women in World War l, op. cit. p. 167 [nous soulignons].

32. Sur cette série, voir Jane Tynan et Suzannah Biernoff, "Making and Remaking the Civilian Soldier: The World War I Photographs of Horace Nicholls», Journal of War and Culture Studies, vol. $5, \mathrm{n}^{\circ} 3$, 2012, p. 277-93 scène élaborée et sur le pouvoir de l'image photographique, cette série était destinée à valoriser l'effort de guerre fourni par les civils.

La photographie en question montre Wood apportant les dernières finitions à un masque, muni d'une palette généreusement couverte de couleurs, attribut de l'artiste par excellence. Dans une pratique qui relève aussi bien de la peinture que du maquillage, l'artiste est représenté en train de peindre un masque posé sur le visage de son client, dont la chair se différencie à peine de la surface du masque, tant la ligne de démarcation entre masque et visage est peu visible. La photographie joue ainsi sur l'illusion que l'artiste peint le client lui-même, suggérant, par extension, que le corps tout entier de ce dernier 
33. David M. Lubin, «Masks, Mutilation, and Modernity: Anna Coleman Ladd and the First World War', Archives of American Art Journal, vol. $47, n^{\circ} 3-4$ (2008), p. 4-15, ici p. 10.

34. Ladd dirigera l'atelier pendant onze mois. Gavin, American Wo men in World War I, op. cit., p. 195.

35. Propos datant de 1948, cités par Bogart et du Louvre, The Plaster Mask as Remedy, op. cit.

36. Sur la formation artistique et la production d'Anna Coleman Ladd avant la guerre, voir Anna Seaton-Schmidt, «Anna Coleman Ladd: Sculptor», Art and Progress, vol. 2, $n^{\circ}$ 9, juillet 1911, p. 251-55.

37. Sur la formation de Wlérick, voir Monique Laurent (dir.), Robert Wlérick. 1882-1944, cat. exp., Musée Rodin/Musée Despiau-Wlérick, 1982, p. 15; sur son œuvre, voir Robert Wlérick. 1882-1944, cat. exp., Musée Despiau-Wlérick, 1991.

38. Sur la formation de Jane Poupelet, voir Charles Kunstler, Jane Poupelet, Paris, 1930; Anne Rivière, "Jane Poupelet ou la beauté dans la simplicité», in Anne Rivière (dir.), Jane Poupelet, 1874-1932. La Beauté dans la simplicité, Paris, 2005 , p. 14-15; 22; 33-34.

39. René Chéruy, «Elèves femmes de Rodin», manuscrit conservé aux archives du musée Rodin, cité par Rivière, «Jane Poupelet», op. cit., pp. $24-25$ serait le fruit de sa création. Nicholls célèbre ici le pouvoir démiurgique d'un artiste capable de redonner vie à ses patients en leur procurant à nouveau un visage acceptable. Il s'agit d'un pouvoir fortement fantasmé, à la hauteur des espoirs déçus relatifs à la capacité de la science médicale d'alors à réparer les mutilations de la face.

Suivant l'exemple de Wood, avec lequel elle entretient une correspondance suivie afin de se familiariser avec ses techniques de fabrication de masques, la sculptrice états-unienne Anna Coleman Ladd, volontaire en service à la Croix-Rouge en Meurthe-et-Moselle, obtient l'aval du ministre de la guerre états-unien pour ouvrir à son tour, toujours dans le cadre de la CroixRouge, un atelier de fabrication de masques à Paris au printemps $1918 . .^{33} \mathrm{En}$ effet, se rendant en décembre 1917 à l'Hôpital du Val-de-Grâce, elle se trouve bouleversée par le spectacle de patients mutilés de la face. Elle décide alors de mettre tout en œuvre pour leur permettre de retrouver un semblant de normalité. ${ }^{34}$

Ladd y fait venir le sculpteur français Robert Wlérick, qui travaillait jusquelà au service de chirurgie faciale du professeur Moure, à Bordeaux, la sculptrice américaine Mary Louise Brent ainsi qu'une sculptrice française, Jane Poupelet. Il n'est guère étonnant que les chirurgiens fassent appel, pour restaurer des corps défigurés, à de tels artistes qui privilégient dans leur travail une facture classique et qui s'attachent à représenter des corps aux proportions harmonieuses et équilibrées. Comme le rapporte malicieusement a contrario le chirurgien Léon Dufourmentel:

Si je devais commander à Picasso un portrait de moi, il me peindrait comme un monstre. Ceci me plairait énormément car cela vaudrait des millions. Mais si jamais Picasso se présentait à moi avec une blessure de la face et que je le transformais en monstre, et bien, je ne suis pas sûr que cela lui plairait autant. ${ }^{35}$

Ladd s'est en effet formée à la sculpture en étudiant les classiques de la statuaire antique à Rome ainsi que les travaux de Rodin. Avant la guerre, elle se fait connaître aux États-Unis par ses sculptures de tritons et de nymphes destinés à décorer fontaines et jardins. ${ }^{36}$ Wlérick étudie la sculpture sur bois à l'École municipale des beaux-arts de Toulouse, où il bénéficie de l'enseignement de Falguière. Il complète ensuite sa formation à Paris, en étudiant avec assiduité les classiques de la sculpture antique au Louvre. Ses travaux se caractérisent par un style classique, dont la grâce est louée aussi bien par Bourdelle que par Despiau. Rodin le compare à cet égard à Donatello. ${ }^{37}$ Quant à Poupelet, elle fut l'une des premières femmes à étudier la sculpture à l'École municipale des beaux-arts et des arts décoratifs de Bordeaux, bénéficiant, dans ce cadre, de cours d'anatomie à la faculté de médecine de la ville (elle y assiste également à des dissections). Entretenant d'étroits rapports amicaux avec Wlérick, elle fait, comme lui, partie du cercle du sculpteur français Lucien Schnegg, attaché à une figuration sereine et dépouillée (dans le sillage de Maillol) et hostile aux expérimentations modernistes de Picasso et de Brancusi. ${ }^{38}$ Poupelet fut par ailleurs probablement l'élève et la praticienne de Rodin, qui la soutint à plusieurs reprises, ainsi que le suggère René Chéruy. ${ }^{39}$

L'atelier de Ladd enregistre très rapidement un vif succès, grâce aux multiples conférences que la sculptrice donne en terrain militaire et hospitalier 
pour faire connaître ses activités, sans parler des nombreux entretiens qu'elle accorde à différents journalistes. Aussi, en novembre 1918, soit moins d'un an après son ouverture, l'atelier a déjà reçu cent vingt commandes de masques pour des soldats français et cinq pour des soldats états-uniens. ${ }^{40} \mathrm{Il}$ arrive à en produire neuf par mois. ${ }^{41}$ C'est une lourde tâche, d'autant que l'atelier fonctionne sur un régime entièrement bénévole. Jane Poupelet y travaille ainsi, par exemple, six après-midis par semaine. ${ }^{42}$ L'étendue de la tâche ne décourage cependant pas les membres de l'atelier, qui comprennent qu'en redonnant aux patients un visage digne de ce nom, ils accomplissent une mission extrêmement importante. Effectivement, de nombreux témoignages font état de la grande reconnaissance qu'éprouvent les anciens patients de l'atelier, ainsi que leur famille, à l'égard d'Anna Coleman Ladd et de son équipe. L'un d'entre eux, le facteur Charles Victor, lui transmet ainsi son immense gratitude en 1920:

Je vous remercient de tous mon cœur de la belle œuvre que vous avez laissez en France, pour nous, pauvres Mutilés de la face, car toute notre vie, nous penseront à tous nos bons amis de la grande Amérique qui ont été ci bon et généreux pour les Mutilés français. ${ }^{43}$

Un autre, le maréchal Marc, lui écrit sans détour que ses prothèses lui ont rendu la vie:

quant à moi je vous en saurai une grande reconnaissance qui dureras toujours et que je ne pourrai pas oublier car je porte et porterai toujours les merveilleux appareils que vous avez conçus c'est grâce à vous que je puis revivre $[. . .]^{44}$

40. Gavin, American Women in World Warl, op. cit., p. 197.

41. Sharon Romm et Judith Zacher, «Anna Coleman Ladd: Maker of Masks for the Facially Mutilated», Plastic and Reconstructive Surgery, vol. 70, $n^{\circ} 1,1982$, p. 104-11, ici p. 104.

42. Sur l'organisation de l'atelier de masques, voir Claudine Mitchell, «L'horreur en face», in Rivière (dir.), Jane Poupelet, op. cit., p. 56-77; Eric Dussourt, «Jane Poupelet, une artiste au service des "Gueules Cassées"», Actes. Société française d'histoire de l'art dentaire, vol. 19, 2014, p. 11-15.

43. Lettre de Charles Victor, facteur à Orphin (Seine-et-Oise) 26 décembre 1920, Anna Coleman Ladd Papers, box 2 , folder 65 , American Red Cross Studio for Portrait-Masks, Smithsonian Archives of American Art, p. 2. [Pour cette lettre comme pour les suivantes, nous avons conservé l'orthographe originelle du texte.] Charles Victor avait été blessé à la face par grenade le 29 septembre 1915, comme il le précise au dos d'une photographie envoyée à Anna Coleman Ladd (Anna Coleman Ladd Papers, op. cit.).

44. Lettre du Maréchal Marc à Anna Coleman Ladd, Castres, le 26 décembre 1920. Anna Coleman Ladd Papers, op. cit., p. 1-2.
Il lui rapporte par ailleurs, dans une autre lettre, qu'il fait la publicité de son travail remarquable chaque fois que l'occasion s'en présente:

J'ai expliqué [aux membres de l'Association castraise des Mutilés] que c'était vous qui avez créé le genre d'appareils que je porte et leur ai fait voir les premiers appareils que vous m'avez fait. Aussi, notre camarade président et tout nos camarades présents à la réunion me prient d'être leurs interprètes auprès de vous pour vous remercier de tout ce que vous avez fait pour nos grands cammarades mutilés et pour moi. [...] En attendant que j'aille vous voir et dire à vos compatriotes tout le bien que vous nous avez fait et tout celui que nous pensons de vous, acceptez, chère Madame Ladd, mes meilleures amitiés. ${ }^{45}$

De tels témoignages ne pouvaient que conforter les membres de l'atelier dans leur action. Une autre dimension doit par ailleurs être prise en compte pour apprécier pleinement la signification spécifique que revêt la fabrication de prothèses faciales pour cette équipe, qui est alors majoritairement composée de femmes. Pour ces dernières, cette activité représente en effet une pratique émancipatoire.

\section{Une pratique d'émancipation pour les femmes}

Que l'atelier soit constitué essentiellement de sculptrices correspond à une situation typique de la Première Guerre mondiale, durant laquelle, pour cause de mobilisation générale des hommes, les femmes accèdent à des responsabilités dont elles étaient jusque-là exclues. Les membres de l'atelier d'Anna Coleman Ladd ont conscience qu'il s'agit d'un moment charnière. Comme le rappelle l'historienne de l'art Claudine Mitchell, Jane Poupelet avait été marquée avant la guerre par les idées d'Hubertine Auclert, grande militante féministe en faveur de l'égalité entre hommes et femmes, qui l'avait orientée vers une pratique sociale de la sculpture explorant des problématiques spécifique- 


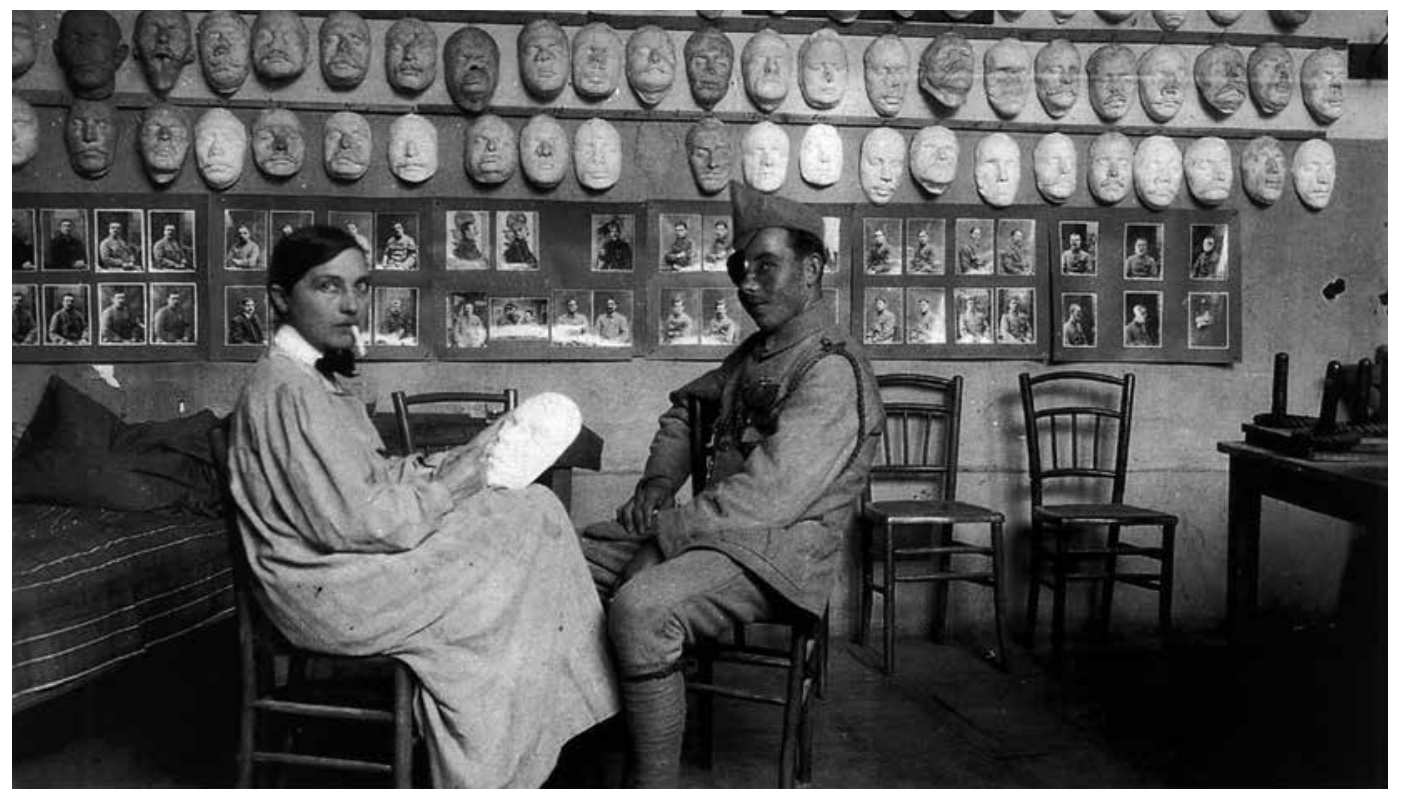

Figure 5. Photographe inconnu, Jane Poupelet dans l'atelier des masques, 1918. Archives Jane Poupelet, collection privée.
45. Lettre du Maréchal Marc à Anna Coleman Ladd, Castres, le 18 mars 1921. Anna Coleman Ladd Papers, op. cit., p. $1-3$

46. Mitchell, «L'horreur en face», op. cit., p. 59 . ment féminines. La sculptrice avait cependant dû renoncer aux thématiques féminines, incompatibles avec un monde de l'art alors essentiellement masculin. ${ }^{46}$ La guerre vient bouleverser la donne, et Poupelet vit une situation inédite dans l'atelier de Ladd, où des hommes mutilés se retrouvent entre les mains d'artistes qui, à l'exception de Wlérick, sont toutes des femmes et dont ils dépendent pour recouvrer un visage acceptable. Les femmes de l'atelier se trouvent ainsi investies d'une importante mission et d'un fort pouvoir. Une photographie, dont l'auteur est inconnu et qui est conservée dans les archives de Jane Poupelet, au Musée de la Piscine, à Roubaix, illustre bien cette transformation à l'œuvre. | fig. 5 |

On y reconnaît Poupelet dans l'atelier avec un patient, en train de travailler à un moulage pour celui-ci. Les murs sont recouverts de photographies et de moulages de blessés ayant bénéficié de prothèses faciales. Les moulages dans la rangée supérieure correspondent au visage dans son état mutilé; les moulages de la rangée inférieure, au visage tel que l'on espère le restaurer. Les photographies sont également organisées selon ce schéma binaire de l'avant et de l'après. Le nombre important de moulages et de photographies accrochés au mur constitue à la fois un témoignage accablant de l'horreur provoquée par la guerre moderne et une preuve de l'effort prodigué par l'atelier pour tenter d'y parer.

L'artiste et le patient regardent tous deux le photographe-et le regardeur-comme pour les prendre à partie de l'importance de l'opération en train de se dérouler. Notons toutefois que, contrairement à d'autres images où la blessure de la face est mise en scène de manière spectaculaire, dans le cas présent, la photographie ne donne guère à voir en détail la blessure du soldat, qui ne semble pas figurer parmi les cas les plus graves de mutilations et qui se dérobe à notre regard grâce au monocle qui la recouvre. 
47. Ce portrait conservé aujourd'hui dans une collection particulière est reproduit dans Rivière (dir.), Jane Poupelet, op. cit., p. 113.

48. Mary Bergstein, «The Artist in His Studio: Photography, Art and the Masculine Mystique», Oxford Art Journal, vol. $18, n^{\circ} 2$, 1995, p. 45-58, ici p. 51-52.

49. Records of the American Red Cross, 1917-1934, File 942.52, "Studio of Portrait Masks», 19171920, National Archives, Washington.

50. Plaza Dinner for the American Women's Assembly, New York, 5 mai 1925, Anna Coleman Ladd Papers, box 2, folder 66, American Red Cross Studio for Portrait-Masks, Smithsonian Archives of American Art.
La photographie opère un bouleversement des codes de représentation traditionnels de l'artiste et de son modèle. La femme ne s'y réduit plus au rôle passif de la muse inspiratrice; occupant désormais la place de l'artiste, elle en hérite de l'attitude active, dont témoigne l'attribut typiquement masculin de la cigarette, que Poupelet tient à la bouche. Cette caractéristique importait probablement à la sculptrice, ainsi que le suggère son Autoportrait à la cigarette, non daté, où elle se représente une cigarette à la bouche, vêtue d'un colet et d'une cravate. ${ }^{47}$ Pour autant, on ne trouve guère ici la dissymétrie radicale entre l'artiste et son modèle telle qu'elle s'incarnera exemplairement dans les portraits de Matisse à l'atelier par Brassaï, où un modèle dénudé et tenant la pose est capturé par un artiste entièrement habillé et tout à son travail. ${ }^{48}$ En effet, il s'agit avant tout d'insister sur la capacité de l'atelier à rendre aux patients une vie sociale. C'est pourquoi le patient masculin apparaît dans une attitude nonchalante, les jambes écartées et souriant légèrement. Sa pose illustre ce qui faisait l'une des caractéristiques de l'atelier, à savoir un climat de bonne humeur et de convivialité. Le personnel de l'atelier se donne pour objectif de faire oublier aux patients leur apparence en les mettant à l'aise et en abolissant ainsi momentanément la distance que leur difformité peut instaurer: "par des après-midis pluvieuses, ils se rassemblaient autour du petit poêle, fumant et chantant, les aveugles jouant aux dominos, les autres aux cartes ou buvant du chocolat, avec toute la gaieté et la "sainte blague" [en français dans le texte] qui étaient les leurs.» ${ }^{49}$ La posture de la sculptrice face à son patient insiste donc ici sur ce lien restauré. De même, alors que généralement, l'économie des regards contribue à l'objectivation du modèle par l'artiste on constate ici une égalité dans le jeu des regards, qui s'adressent tous deux au spectateur. De la sorte, en mettant en scène l'accès des femmes à des positions et statuts jusque-là traditionnellement occupés par des hommes, cette photographie témoigne bel et bien d'un bouleversement en cours et entend ainsi œuvrer à la reconnaissance que la société doit accorder aux femmes à cet égard.

Lorsque l'après-guerre balaiera les espoirs des féministes en restaurant l'ordre entre les sexes d'avant 14-18, le souvenir du rôle des femmes à l'atelier des masques conduira Anna Coleman Ladd à en appeler à la nécessité, pour les femmes, de s'affirmer comme artistes et de prendre la place qui leur revient dans l'histoire de l'art. Elle affirme ainsi en 1925, lors d'un dîner de l'American Women's Assembly:

Bien que les femmes hantent les galeries, les salles de conférences, les écoles d'art, ce sont les hommes qui, par le passé, ont été les plus grands collectionneurs d'art et les plus grands artistes. Les femmes d'aujourd'hui changeront peut-être cela, mais nous avons encore une longue route à faire et un lourd handicap. ${ }^{50}$

\section{Les masques, une duperie?}

Malgré leur succès apparent, il ne faudrait pas surestimer l'efficacité de ces masques au quotidien. Certes, à l'instar des chirurgiens plastiques maxillofaciaux, les ateliers de masques vantent la qualité de leurs produits à l'aide de contrastes qui opposent de façon éloquente l'aspect du patient blessé sans masque et avec masque. | fig. 6a et 6b | Cette rhétorique culmine dans l'un des films publicitaires produits par l'atelier de Ladd, entièrement construit 

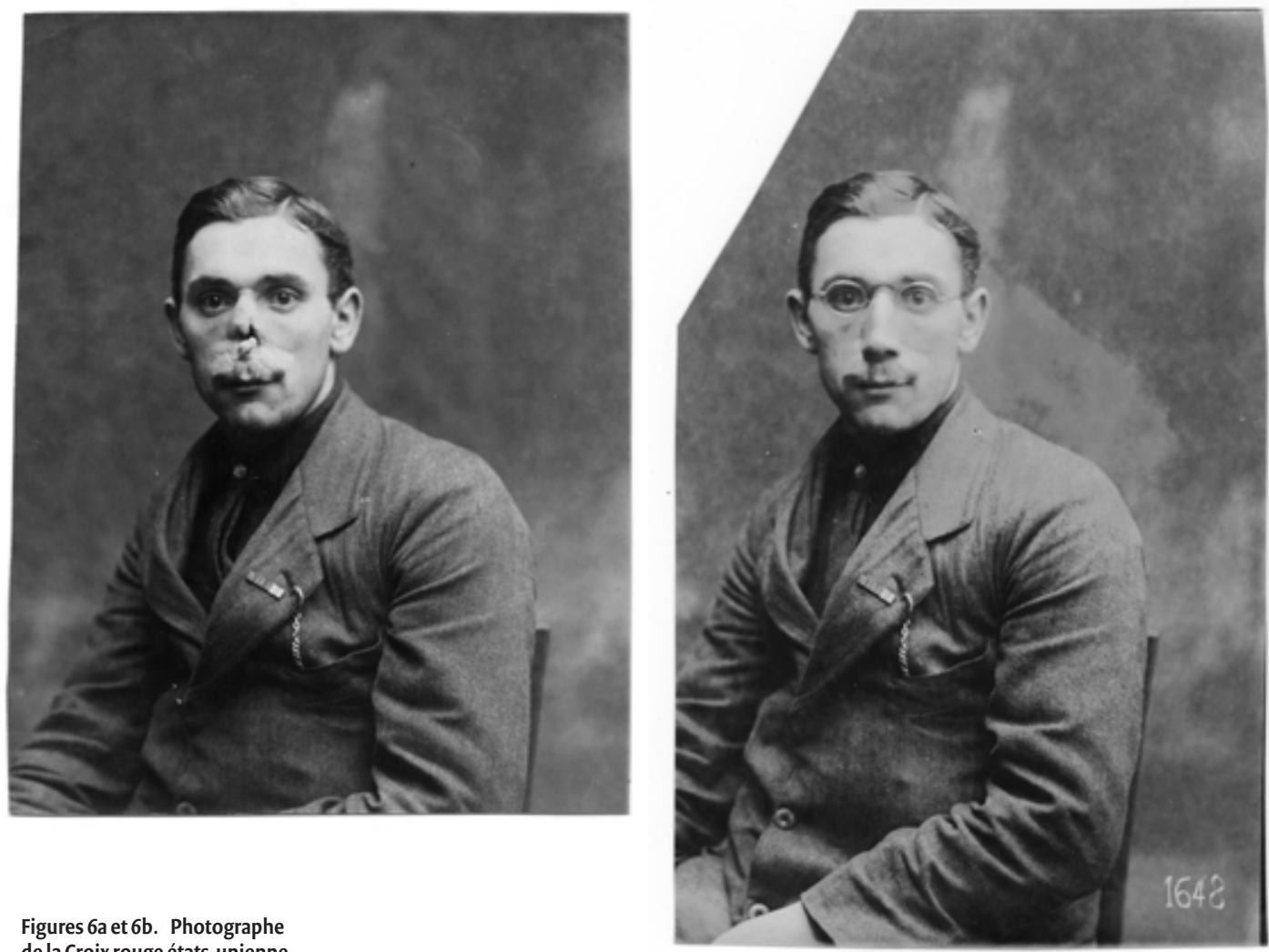

Figures $6 a$ et $6 b$. Photographe de la Croix rouge états-unienne, Soldier facial reconstruction documentation photographs, vers 1918. Anna Coleman Ladd papers, circa 1881-1950. Archives of American Art, Smithsonian Institution.
51. Film visionnable sur le site des Smithsonian Archives of American Art: www.smithsonianmag.com/videos/category/history/ about-face/.

52. Delaporte, Gueules cassées, op. cit., p. 121-22; Katherine Feo, "Memory, Masks and Masculinities in the Great War», Journal of Design History, vol. 20, $\mathrm{n}^{\circ} 1$, printemps 2007, p. 17-27, ici p. 23-25. sur un effet de surprise: l'on y voit des hommes d'apparence absolument normale retirer tout à coup des morceaux de leur visage, révélant ainsi qu'il s'agit de prothèses. Rien n'aurait pu le laisser deviner à l'avance, tant la différence entre la chair et le masque y est imperceptible. ${ }^{51}$ Mais cette indétermination entre la peau et le métal doit autant aux propriétés du médium filmique qu'à l'habileté des sculpteurs; elle est obtenue à la fois grâce à la distance nécessaire aux prises de vue, à l'organisation séquentielle du film ainsi qu'à la pellicule en noir et blanc, qui estompe les différences de carnation.

La réalité n'est pas aussi miraculeuse. Tout d'abord, d'un point de vue pratique, les masques et prothèses présentaient tous des inconvénients, qu'ils soient réalisés en gélatine, en plastique, en vulcanite ou en métal peint. Ils pouvaient perdre très rapidement leurs coloris d'origine, induisant un écart humiliant entre le teint du masque et celui du visage. Ils devaient être souvent restaurés, voire remplacés. Enfin, le port d'éléments réalisés en cuivre ou comportant de la colle pouvait causer des irritations cutanées sévères, sans parler du désagrément dû à leur poids. C'est pourquoi l'enthousiasme engendré par les prothèses pouvait parfois laisser rapidement place à la déception et à la résignation, le mutilé se résolvant à arborer son visage tel quel. Les historiennes Sophie Delaporte et Katherine Feo considèrent ainsi que le recours aux prothèses faciales a constitué en définitive une solution relativement marginale. ${ }^{52}$ 
53. Lettre d'Oliver C. Small à Anna Coleman Ladd, 31 mars 1919, Box 2, folder 65, American Red Cross Studio for Portrait-Masks, Smithsonian Archives of American Art.

54. Cet aspect a été bien détaillé par Feo, «Memory, Masks and Masculinities», op. cit.

55. Comme le rappellent Geneviève Allard et Pierre Lefort, avant quelque autre fonction que ce soit, le masque commence toujours par dissimuler. Geneviève Allard et Pierre Lefort, Le Masque, Paris, 1984, p. 19.

56. Debra Lennard, «Censored Flesh: The Wounded Body as Unrepresentable in the Art of the First World War», The British Art Journal, vol. $12, n^{\circ} 2$, automne 2011, p. 2233 , ici p. 30

57. Sur l'ambivalence du masque, voir Odette Aslan, «Du rite au jeu masqué», in Odette Aslan et Denis Bablet (dir.), Le Masque, du rituel au théâtre, Paris, 1985, p. 281-82.

58. Allard et Lefort, Le Masque, op. cit., p. 48
Indépendamment même de ces questions techniques, le port des masques n'allait pas sans difficulté. Tout d'abord, il est évident que la fixité des traits des masques devait éveiller un sentiment perturbant au sein de l'entourage du blessé. En effet, le masque ne pouvait, comme un visage véritable, traduire les émotions et les réactions de son porteur en expressions faciales. Anna Coleman Ladd reçoit à cet égard une lettre en 1919 d'un électricien, qui lui demande de l'aide car sa prothèse nasale ne «s'adapte pas aux traits du visage comme elle le devrait». ${ }^{53}$

Une telle immobilité du siège de l'identité qu'est le visage marquait donc irrémédiablement le porteur du masque d'un sceau d'inquiétante étrangeté qui devait engendrer malaise et rejet auprès de ceux qu'il rencontrait et côtoyait. ${ }^{54}$ Opérant une rencontre entre la chair et le métal, le masque brouillait les repères et faisait basculer son propriétaire dans la sphère de l'inhumain. En outre, le fait de découvrir un masque aux traits rigides à la place d'un visage à la physionomie mobile devait induire un sentiment de morbidité, l'impression d'avoir affaire à un être mort. La prothèse faciale, supposée redonner vie à son propriétaire, pouvait alors basculer du côté du masque mortuaire, avec toutes les implications sinistres que cela suppose.

Surtout, une fois le masque identifié comme tel, impossible pour lui de ne pas renvoyer à l'une de ses fonctions, qui est de dissimuler. ${ }^{55}$ Se pose alors nécessairement la question de savoir ce qu'il cherche à occulter et de découvrir ce qui exige d'être ainsi dérobé au regard. Comme l'écrit Debra Lennard, "le non visible exige d'être vu». ${ }^{56}$ La réponse que l'imagination apporte à cette interrogation peut être tout aussi effrayante, sinon plus, que la vision de la blessure elle-même. Par nature ambivalent et duplice, le masque oscille ainsi toujours entre révélation et dissimulation, fonctionnant, dans le cas des blessés de la face, comme le marqueur d'une perte irrémédiable. ${ }^{57}$

Pour le mutilé lui-même, le recours à un masque n'avait rien d'une opération anodine. Généralement, lorsque l'on enfile un masque, c'est pour endosser une deuxième peau, une autre identité que la sienne; c'est pour cesser momentanément d'être soi. ${ }^{58}$ En effet, le masque, tout en dissimulant, métamorphose. Qui dit masque dit travestissement et donc démultiplication et transformation de l'identité. Dans le cas des blessés de la face, cette identité polysémique devient d'autant plus complexe qu'il leur faut, autant que possible, se déguiser en permanence là où, en principe, le port du masque est limité dans le temps, qu'il s'agisse de représentation théâtrale ou de rituel performé. En outre, les blessés de la face représentent un cas particulier de travestissement: il leur faut porter un masque pour redevenir un soi-même qu'ils ne sont plus, et qu'ils ne seront jamais plus. On peut imaginer le désarroi et le vertige induits par la nécessité d'enfiler une réplique de soi-qui plus est imparfaite et artificielle-pour tenter de retrouver un soi qui n'existe plus, et d'échapper ainsi à un soi existant dans lequel on ne se reconnaît guère.

Le mutilé sait bien que le visage offert par le masque, de par sa nature artificielle, ne peut jamais offrir qu'un simulacre d'identité. Or du simulacre au mensonge et à la supercherie, il n'y a qu'un pas. En effet, bien qu'on ne puisse mettre en doute les intentions bienveillantes et altruistes des auteurs de prothèses faciales, il faut garder à l'esprit que l'enjeu relatif au camouflage des blessés de la face ne se limite pas à la sphère individuelle du mieux-être des 


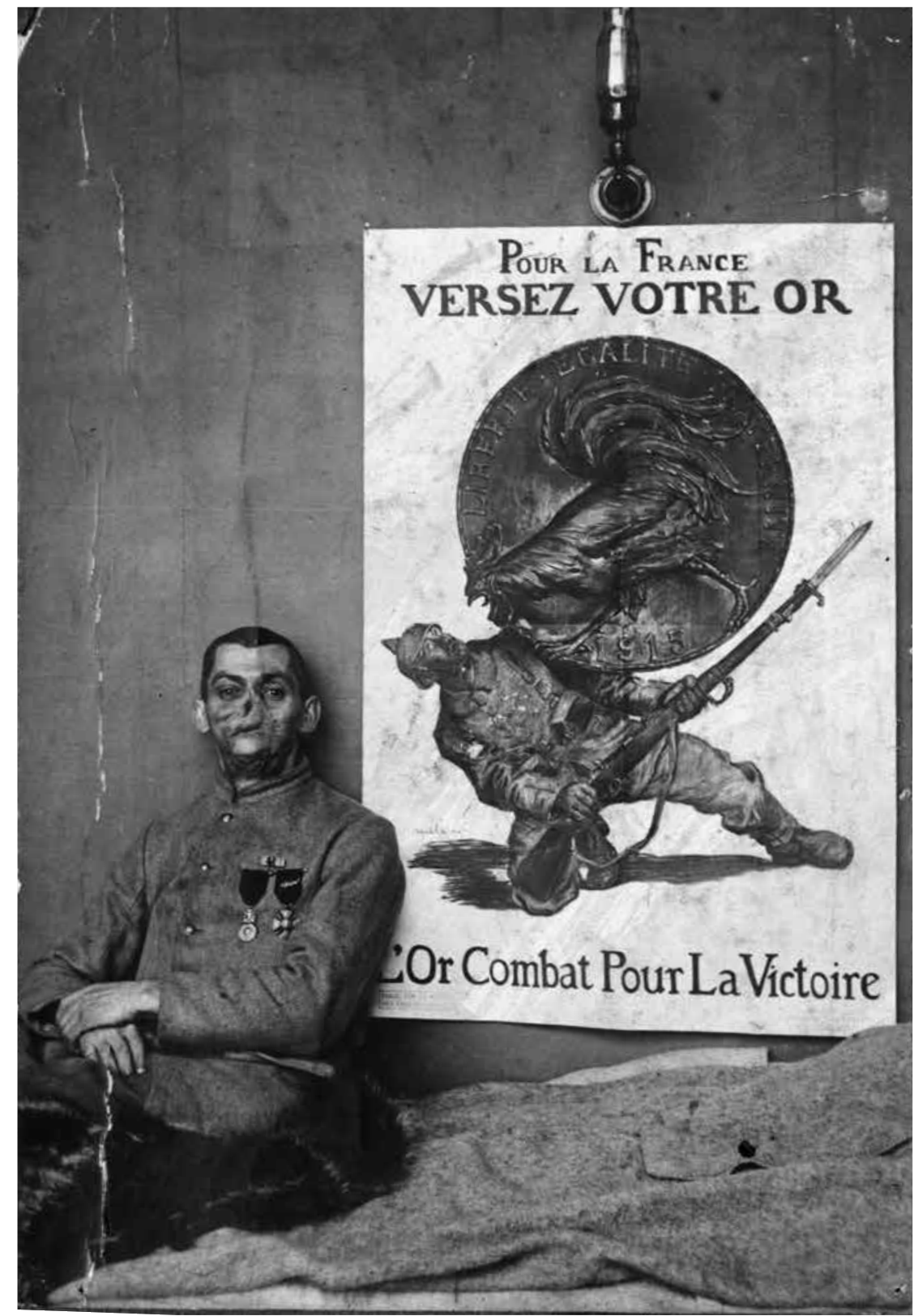

Figure 7. Photographe de

la Croix rouge états-unienne,

Soldier facial reconstruction

documentation photograph,

vers 1920. Anna Coleman Ladd

papers, circa 1881-1950. Archives

of American Art, Smithsonian

Institution. 
Figure 8. Photographie tirée de Ernst Friedrich, Guerreà la guerre, 1924. Accompagnée de la légende suivante, écrite en quatre langues: Le traitemenwt d'eaux minérales des prolétaires: presque la figure entière arrachée. Photo: avec l'amabilité de la Osler Library of the History of Medicine, Université McGill, Montréal.
59. Feo, «Memory, Masks and Masculinities», op. cit., p. 20.

60. Lennard, «Censored Flesh», op. cit., p. 27-28; Biernoff, «The Face of War», op. cit., p. 43.

61. Sander L. Gilman, Making the Body Beautiful: A Cultural History of Aesthetic Surgery, Princeton, 1999, p. 161.

62. Suzannah Biernoff, «The Rhetoric of Disfigurement in First World War Britain», Social History of Medicine, vol. $24, n^{\circ} 3$, p. 666-85, ici p. 669.

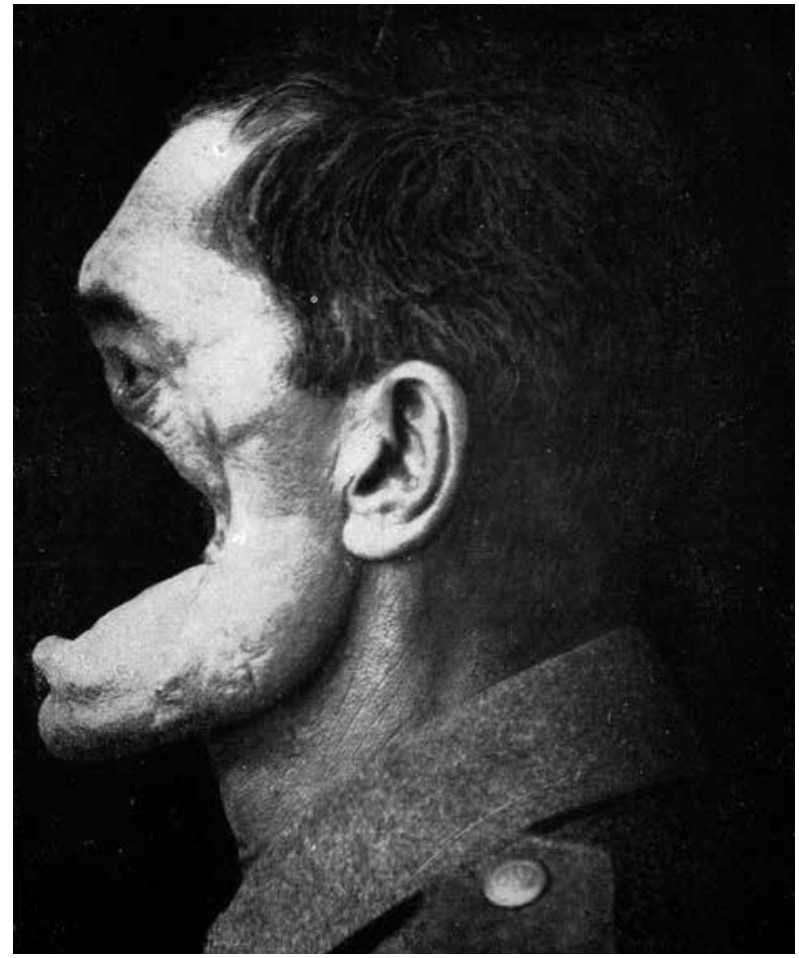

mutilés mais relève également de problématiques concernant la société toute entière. Les chirurgiens plastiques défendaient déjà leur pratique de la réparation faciale avec des arguments économiques et sociaux en expliquant que rendre aux mutilés un visage acceptable leur permettrait de retrouver un emploi et d'éviter par conséquent d'être à la charge de la société, tout en offrant à cette dernière une main-d'œuvre non négligeable et fort bienvenue après l'hécatombe. ${ }^{59}$ Selon une logique similaire, les fabricants de masques contribuent eux aussi à la cohésion économique et sociale.

Le visage mutilé est "dangereux à voir», "tabou», parce qu'il montre sans détour l'horreur de la guerre, une horreur sans précédent, due au caractère industriel du conflit. ${ }^{\circ}$ Le blessé de la face arbore de manière trop perturbante qu'il a sacrifié pour la Nation une partie de lui-même que celle-là est totalement incapable de lui restituer, à savoir son identité et son humanité. C'est pour cette raison, notamment, que les blessés de la face sont déclarés inaptes au front, de crainte que leur apparence ne démoralise les troupes. ${ }^{61}$ Il est par conséquent primordial de dissimuler autant que possible ces blessés, contrairement aux autres types de mutilés et d'amputés, quant à eux largement représentés comme tels et célébrés comme des héros de guerre dans la presse, affiches et autres médias. ${ }^{62}$ La mise en place de masques destinés à camoufler les blessures doit dès lors être interprétée comme faisant partie d'un dispositif de censure élargi, qui vise à faire disparaître, autant que possible, les visages mutilés du champ de vision. Il s'agit, avec les masques, de faire écran, de gommer une réalité dérangeante et menaçante 
63. Ibid., p. 681.

64. Anna Coleman Ladd Papers, box 3, folder 2, American Red Cross Studio for Portrait-Masks, File: Photographs 1914-1925 Smithsonian Archives of American Art.

65. Ernst Friedrich, Krieg dem Kriege! Guerre à la Guerre! War Against War! Oorlog aan den 0orlog!, Berlin, 1924. Voir à ce sujet Dora Apel, "Cultural Battlegrounds: Weimar Photographic Narratives of War», New German Critique, $n^{\circ} 76$, hiver 1999, p. 49-84, ici p. 52-62; Susan Sontag, Regarding the Pain of Others, New York, 2003, p. 14-15.

66. Friedrich, Krieg dem Kriege, op. cit., p. 217. pour l'ordre social. Enfiler un masque revient ainsi, pour son propriétaire, à contracter un pacte par lequel il s'engage et consent à ne pas troubler l'ordre en dissimulant sa chair meurtrie. ${ }^{63}$

Les fabricants de masques avaient bien conscience de cet enjeu. En témoigne une photographie conservée dans les archives d'Anna Coleman Ladd, qui met en scène de manière éclatante la charge subversive que représentent les gueules cassées à l'égard de la société et des discours officiels sur la guerre. Un soldat au visage gravement mutilé se trouve près d'une affiche comportant un appel à souscription qui circula massivement pendant la guerre. ${ }^{64}$ | fig. 7 | On y lit: «l'Or combat pour la Victoire». La terrible blessure du soldat vient vider de tout son sens le terme de victoire, et offre un démenti sans appel à la propagande militaire officielle véhiculée par l'affiche. Dans ce contexte, chercher à camoufler les blessures revient à vouloir occulter ce qui met à mal les positions officielles et qui exhibe de manière trop irréfutable l'inanité de certaines croyances et valeurs. La fabrication de masques peut à cet égard être a posteriori interprétée comme l'un des rouages d'une vaste campagne mensongère cherchant à minimiser et à nier la violence et la brutalité de la guerre.

Il n'est guère surprenant, dans cette perspective, que certains militants pacifistes aient délibérément choisi après la guerre de montrer des gueules cassées, dans toute leur crudité, afin de s'en prendre à la société et aux valeurs qui permirent qu'un tel conflit eut lieu et surtout, pour éviter qu'une telle catastrophe ne se reproduise. Il s'agit en effet littéralement de faire tomber les masques et de donner à voir «la véritable face de cette guerre naturaliste, désenchanteresse, révoltante». Ce sont en effet les propos de l'anarchiste pacifiste allemand Ernst Friedrich, lorsqu'il publie en 1924 son manifeste antimilitariste Guerre à la guerre, qui fait alors fortement sensation. ${ }^{65}$ Parmi ses nombreuses illustrations provenant, pour la plupart, de matériaux iconographiques censurés pendant la guerre, figurent vingt-trois portraits de blessés de la face pris en charge par la clinique du Dr Jacques Joseph à Berlin. | fig. 8| Les portraits sont accompagnés de légendes violemment antiphrastiques, rédigées en quatre langues. Par exemple, à la formule du maréchal von Hindenburg «La guerre est pour moi comme un traitement à l'eau minérale», Friedrich associe une photographie de gueule cassée qu'il légende ainsi: «Le traitement d'eaux minérales des prolétaires: la figure presque entière arrachée». ${ }^{66} \mathrm{Il}$ s'agit, par la force du montage orchestrant de puissants chocs entre les textes et les images ainsi qu'entre les textes euxmêmes, de démonter une rhétorique nationaliste belliqueuse aussi mensongère que dangereuse, de la mettre à nu.

Quel meilleur porte-parole, en effet, contre la barbarie et l'inhumanité de la guerre, que ces visages tristement dévastés et comme vidés d'euxmêmes? L'ouvrage de Friedrich enjoint ainsi le lecteur à cesser de se laisser abuser par tout leurre, par toute supercherie au sujet de la guerre-ce qui implique, entre autres, de chercher à voir par-delà les masques portés par les gueules cassées. 